\title{
Insight into the Charge Density Wave Gap from Contrast Inversion in Topographic STM Images
}

\author{
M. Spera $\odot,{ }^{1, \dagger}$ A. Scarfato๑,$^{1, \dagger}$ Á. Pásztor $\odot,{ }^{1}$ E. Giannini $\odot,{ }^{1}$ D. R. Bowler $\odot,{ }^{2}$ and Ch. Renner $\oplus^{1, *}$ \\ ${ }^{1}$ Department of Quantum Matter Physics, University of Geneva, 24 Quai Ernest-Ansermet, CH-1211 Geneva 4, Switzerland \\ ${ }^{2}$ London Centre for Nanotechnology and Department of Physics and Astronomy, University College London, \\ London WCIE 6BT, United Kingdom
}

(Received 29 June 2020; revised 23 September 2020; accepted 1 December 2020; published 31 December 2020)

\begin{abstract}
Charge density waves (CDWs) are understood in great detail in one dimension, but they remain largely enigmatic in two-dimensional systems. In particular, numerous aspects of the associated energy gap and the formation mechanism are not fully understood. Two long-standing riddles are the amplitude and position of the CDW gap with respect to the Fermi level $\left(E_{F}\right)$ and the frequent absence of CDW contrast inversion $(\mathrm{CI})$ between opposite bias scanning tunneling microscopy (STM) images. Here, we find compelling evidence that these two issues are intimately related. Combining density functional theory and STM to analyze the CDW pattern and modulation amplitude in $1 T$-TiSe ${ }_{2}$, we find that CI takes place at an unexpected negative sample bias because the CDW gap opens away from $E_{F}$, deep inside the valence band. This bias becomes increasingly negative as the CDW gap shifts to higher binding energy with electron doping. This study shows the importance of CI in STM images to identify periodic modulations with a CDW and to gain valuable insight into the CDW gap, whose measurement is notoriously controversial.
\end{abstract}

DOI: 10.1103/PhysRevLett.125.267603

Introduction.-The charge density wave (CDW) ground state is an atomic length scale periodic modulation, combining lattice and charge degrees of freedom [1]. The precise mechanism driving this phase transition remains largely unknown. Fermi surface nesting, electron-electron or electron-phonon interactions, and coupling of electrons to other degrees of freedom in the host crystal are among the main mechanisms discussed over the years $[2,3]$.

Below the CDW phase transition temperature, atoms rearrange into periodic lattice distortions. Concomitantly, charge is redistributed in real space to form alternating regions of charge accumulation and charge depletion. In the classic Peierls mechanism, mostly states in the vicinity of the Fermi level $\left(E_{F}\right)$ are involved in the CDW formation and a gap opens at $E_{F}$.

Scanning tunneling microscopy (STM), owing to its high spatial topographic resolution, is an ideal probe to characterize the real space charge ordering. In the Peierls scenario, constant current STM images of the CDW depend on sample bias voltage $\left(V_{b}\right)$ polarity: negative bias will show enhanced intensity over charge accumulation regions, whereas images of the same area at positive bias will show

Published by the American Physical Society under the terms of the Creative Commons Attribution 4.0 International license. Further distribution of this work must maintain attribution to the author(s) and the published article's title, journal citation, and DOI. enhanced intensity over charge depleted regions. This is known as contrast inversion (CI) of the CDW STM pattern.

Contrast inversion is often considered a hallmark of the CDW contribution to the STM topographic signal. However, clear CI between opposite polarity STM images has only been reported in very rare cases [4,5], including high temperature superconductors [6] and transition metal dichalcogenides [7]. In a thorough theoretical analysis for $2 \mathrm{H}$ - $\mathrm{NbSe}_{2}$, Sacks et al. [8] conclude that CI does not take place in this material due to band structure effects. They further contend that $\mathrm{CI}$ is in general not expected for twodimensional CDW systems. In a more recent STM study of $\mathrm{TaS}_{2}, \mathrm{TaSe}_{2}$ and $\mathrm{NbSe}_{2}$, Dai et al. [9] conclude that strong lattice distortions completely mask possible CI arising from electronic contributions to the CDW amplitude in the topography.

In addition to the real space reconstruction introduced above, the CDW ground state is also characterized by a gap in the electronic density of states (DOS). For strong coupling CDW materials the gap does in general not open for all momenta or necessarily at the Fermi level $[10,11]$. In particular for $\mathrm{TiSe}_{2}$, the Ti $3 d_{z^{2}}$ and the $\mathrm{Se} 4 p_{z}$ bands only marginally participate in the CDW reconstruction [12-14]. Moreover, the electronic nature of this compound is still a matter of debate. For instance, recent angle-resolved photoemission spectroscopy (ARPES) studies find either semiconducting [13] or semimetallic [14] behavior with the bands affected differently upon the CDW transition.

Since the CDW gap opens only in a small portion of the band structure and scanning tunneling spectroscopy (STS) 
is a momentum averaging technique, STS spectra lack distinct spectroscopic features allowing a sure identification of a momentum dependent CDW gap. Moreover, clear disentanglement of CDW related reductions of the DOS from band structure features are further complicated in $\mathrm{TiSe}_{2}$ due to its debated electronic nature. Therefore, determining the amplitude and position of the CDW gap with respect to $E_{F}$ by STS is problematic. Here, instead of spectroscopic data, we focus on bias dependent topographic images, which we demonstrate to provide novel insight into the CDW gap and contrast inversion. Supported by DFT simulations and a simple one-dimensional model, topographic images show that the absence of $\mathrm{CI}$ in opposite polarity STM images is a direct consequence of the CDW gap not opening at $E_{F}$. When present, CI informs about the CDW gap amplitude, which is often significantly larger than expected from the phase transition temperature. Finally, detailed analysis of CI provides direct clues about the inadequacy of Fermi surface nesting as the main mechanism driving the CDW phase transition.

Results and discussion.-Simple visual inspection of the constant current STM images presented in Fig. 1 (top row) shows that CDW contrast inversion does not happen between the opposite polarities data, but between the frames acquired at -300 and $-100 \mathrm{mV}$. These images acquired at different bias voltages were aligned with atomic

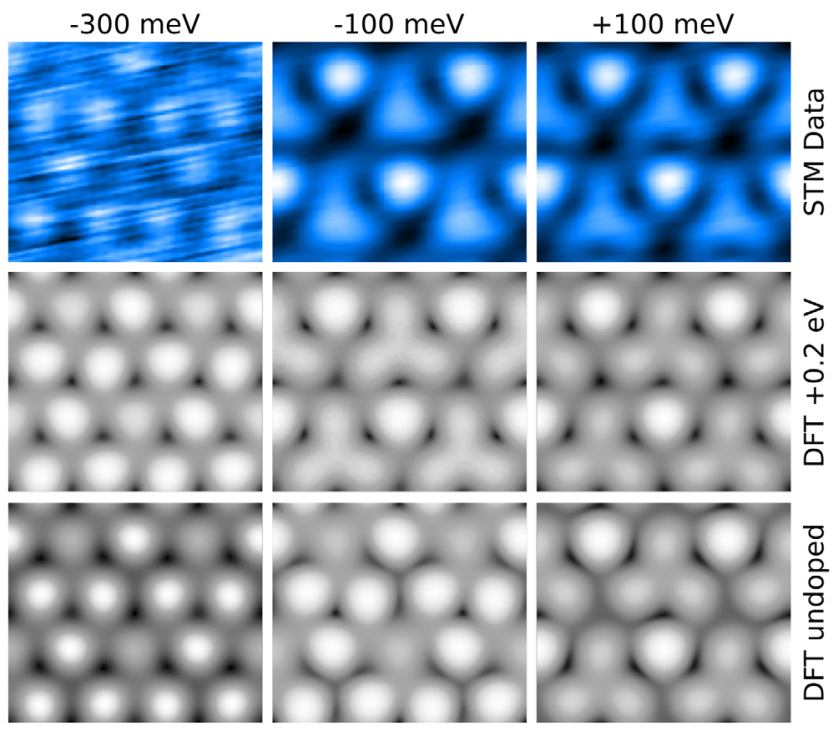

FIG. 1. Charge order contrast inversion revealed by STM on $1 T$-TiSe ${ }_{2}$. Top row: $1.4 \times 1.2 \mathrm{~nm}^{2}$ bias dependent STM micrographs of the same area showing contrast inversion below the Fermi energy between the two images recorded at $V_{b}=$ $-300 \mathrm{mV}$ and $V_{b}=-100 \mathrm{mV}$. Set parameters of STM data are, from left to right, $V_{b}=-300 \mathrm{mV}$ and $I_{t}=600 \mathrm{pA}, V_{b}=$ $-100 \mathrm{mV}$ and $I_{t}=100 \mathrm{pA}, V_{b}=100 \mathrm{mV}$ and $I_{t}=200 \mathrm{pA}$. The $Z$ range of the STM data from left to right is 4.3, 37.2, and $36.1 \mathrm{pm}$. Middle and bottom rows show the DFT simulations of the expected STM topographic contrast as a function of bias in electron doped and in pristine $1 T-\mathrm{TiSe}_{2}$, respectively. scale precision using well-identified single atom oxygen and titanium defects resolved in large-scale images [15]. In contradiction to these experimental findings, detailed DFT calculations for undoped pristine $1 T-\mathrm{TiSe}_{2}$ predict $\mathrm{CDW}$ contrast inversion between -100 and $+100 \mathrm{mV}$ (Fig. 1, bottom row), as expected in the classic Peierls model.

DFT does reproduce the experimental observation when doping electrons into the unit cell (Fig. 1, middle row). The energy where CI takes place in this case depends on carrier concentration, shifting to higher binding energy with increasing electron content. Interestingly, there is a practical limit as to how far CI can be observed at negative bias (see Supplemental Material Sec. II for extended DFT data [16]). Indeed, for high enough doping, CDW contrast disappears altogether before the negative bias voltage where contrast inversion would actually appear.

The STM data and DFT simulations of Fig. 1 reveal a remarkable doping dependence of the energy where CI occurs. The disagreement between experiment and DFT for undoped $\mathrm{TiSe}_{2}$ is likely to arise from two different sources: first, the well-known problem with DFT underestimating band gaps means that the fine details of the $\mathrm{TiSe}_{2}$ gap will not be perfectly accurate; second, the well-known selfdoping of $\mathrm{TiSe}_{2}$ with excess $\mathrm{Ti}$ in experiments, which will shift the Fermi level. Bias dependent STM images of the CDW near single atom defects also suggest a doping dependence of the CI. Most of the area imaged in Fig. 2 does not show any CI between -100 and $+100 \mathrm{mV}$, as already pointed out in Fig. 1. However, there is contrast inversion between these two biases in the right-hand side region of Figs. 2(e) and 2(f), between the defects marked $A$ and $B$. This region with inverted contrast is expanding with increasing negative sample bias to encompass most of the field of view at $-300 \mathrm{mV}$ [Figs. 2(a)-2(d)].

Comparing with DFT simulations, the absence of CI at opposite biases in the central regions of Fig. 2, away from the defects, is consistent with a globally electron-doped system. The contrast around the defects indicates that the doping is locally modified: while defects $C$ and $D$ behave markedly as electron donors, the presence of CI near zero bias in the region between defects $A$ and $B$ suggests that they have a light hole-doping character, such that their combined action turns the area towards neutrality. The result is an increasing electron doping gradient from defects $A$ and $B$ to defects $C$ and $D$, with the strongest electron doping character around defect $D$ [27]. The role of defects and bias dependent STM imaging has been discussed previously in $2 \mathrm{H}-\mathrm{NbSe}_{2}$ [28]. However, the focus of that study was on the ability of defects to stabilize the CDW phase near the transition temperature without addressing $\mathrm{CI}$ and local doping effects. Note that while we observe a marked spatial dependence of the CI due to local doping, the corresponding shift of the STS spectra is very small (see Supplemental Material Fig. S2 for additional STS data [16]). 


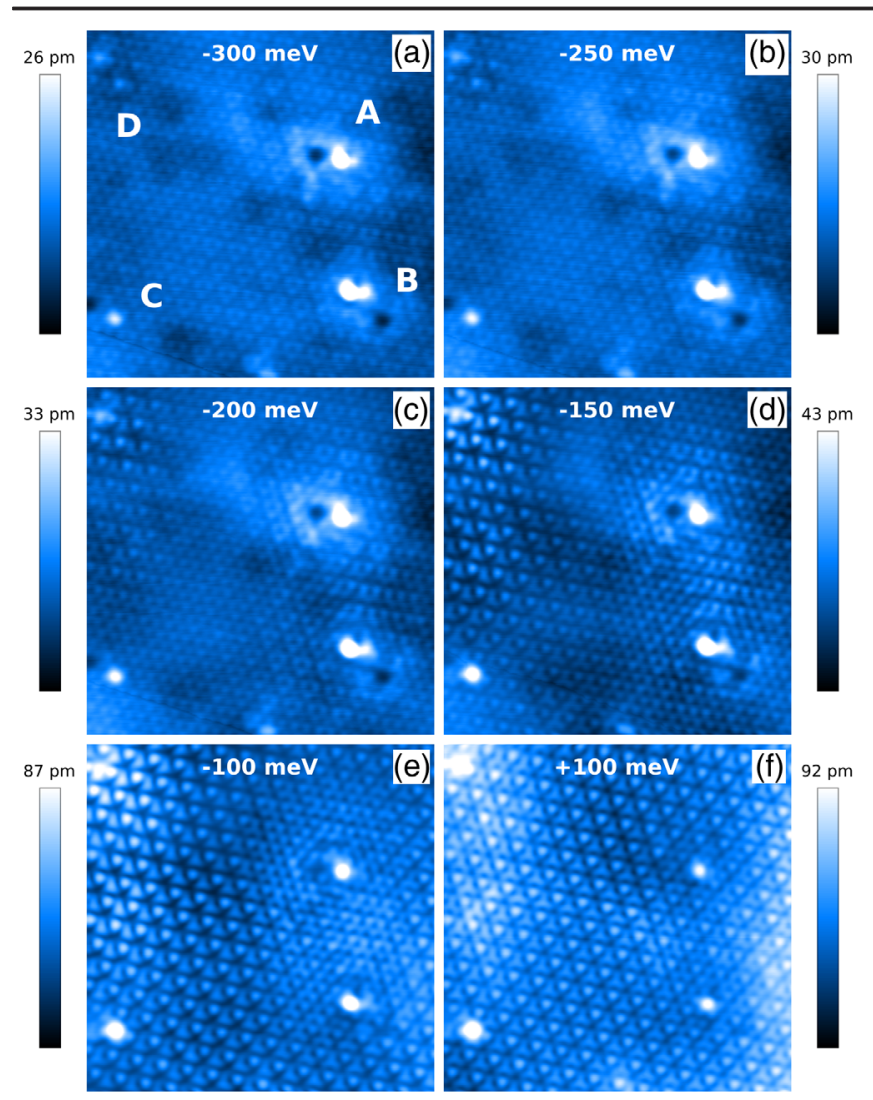

FIG. 2. Doping dependence of the CDW contrast inversion. $10 \times 10 \mathrm{~nm}^{2}$ STM topography of $1 T-\mathrm{TiSe}_{2}$ at (a) $-300 \mathrm{mV}$, (b) $-250 \mathrm{mV}$, (c) $-200 \mathrm{mV}$, (d) $-150 \mathrm{mV}$, (e) $-100 \mathrm{mV}$, and (f) $+100 \mathrm{mV}$ sample bias. CI happens at an increasingly higher binding energy near defects $A$ to $D$, indicating their different doping nature (see text and Fig. S3 in Supplemental Material, Sec IV [16]). Set currents are (a) 600 pA; (b) 300 pA; (c),(d),(f) $200 \mathrm{pA}$; (e) $100 \mathrm{pA}$.

As mentioned in the introduction, there are very few topographic STM studies reporting contrast inversion for any CDW material in the literature $[4,9,29]$. In most cases, the focus has been on comparing images taken at opposite polarities, as would be expected in a classic Peierls transition. Here, we find that images taken at low opposite biases are indeed very similar, while clear CI is observed between images measured at selected negative biases. In the following, we demonstrate that this observation is a direct consequence of a CDW gap opening below $E_{F}$ (corresponding to $V_{b}=0 \mathrm{~V}$ ) and shifting to higher binding energy with increasing electron doping.

First, we demonstrate that the observed contrast inversion in the STM micrographs is indeed a CDW feature. To this end, we consider two real space images of the CDW taken at two different biases below $E_{F}$. Figures 3(a) and 3(b) are magnifications from Figs. 2(b) and 2(e) of the uniform region in between the defects $A-D$, where we decided to keep defect $A$, visible in the top right corner of the cropped images, as a reference point. Figure 3(a)

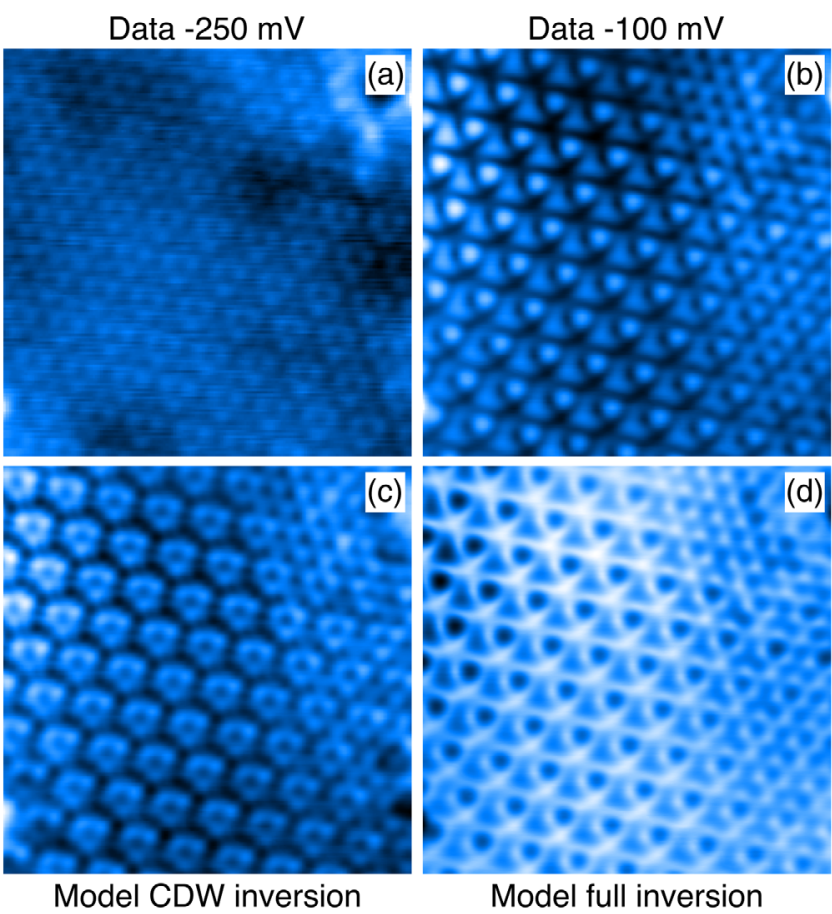

FIG. 3. Charge order origin of the contrast inversion observed in bias dependent STM images of $1 T$-TiSe ${ }_{2}$. (a) Negative sample bias image at $-250 \mathrm{mV}$ showing the real space distribution of charge accumulation (set current is $300 \mathrm{pA}, z$ range $=33 \mathrm{pm}$ ). (b) Negative sample bias image at $-100 \mathrm{mV}$ showing the real space distribution of charge depletion (set current is $100 \mathrm{pA}, z$ range $=66 \mathrm{pm}$ ). (c) Contrast inversion applied only to the CDW component of the image in (b). (d) Contrast inversion applied to all components of the image in (b). Image size is $6 \times 6 \mathrm{~nm}^{2}$.

was acquired at a large negative bias, where the periodic pattern reflects the charge accumulation associated with the CDW reconstruction in $\mathrm{TiSe}_{2}$. Figure 3(b) was acquired at a smaller negative bias closer to the Fermi level, and shows the corresponding periodic pattern of charge depletion.

If the differences between the two images in Figs. 3(a) and 3(b) are due to the CDW, they should be related through an inversion of the periodic charge order reconstruction pattern. To verify this, we numerically invert the contrast of the CDW signal of the charge depletion image in Fig. 3(b) in the following way. First, we apply Fourier filtering to separate the signal corresponding to the CDW from the rest of the image. We then invert this CDW image and recombine the result with the rest of the original micrograph (see Supplemental Material Sec. V for more details [16]). The resulting image shown in Fig. 3(c) is in excellent agreement with the experimental charge accumulation image in Fig. 3(a). Alternatively, if we invert not only the CDW component, but the full image, including the atomic lattice contrast in Fig. 3(b), we obtain a completely different topographic pattern unable to reproduce the experimental one [Fig. 3(d)]. The contrast 
difference between the two STM topographies in Figs. 3(a) and 3(b) is thus definitely due to the CDW contrast inversion.

An interesting aspect of the STM images in Figs. 2(e)-2(f) is that we not only observe the same CDW pattern at $\pm 100 \mathrm{mV}$ opposite polarities in some regions, they also show the same CDW modulation amplitude defined as the peak-to-peak amplitude of the local CDW topographic signal (see Sec. VI in the Supplemental Material for the technical details of the analysis [16]). Such close correspondence (both in phase and amplitude) between opposite polarity topography is not possible if the CDW gap opens across the Fermi level as we show in the simple onedimensional model illustrated in Fig. 4.

Let us consider a one-dimensional (1D) BCS-like local density of states (DOS) [9], with a partial gap centered at $E_{F}$ [Fig. 4(c)] and a harmonic spatial modulation where the states above and below the gap midpoint are spatially $180^{\circ}$ out of phase [Fig. 4(a)]. A constant current STM image amounts to integrating over all states from $E_{F}$ up to the imaging bias at each sampling point. Similarly, we
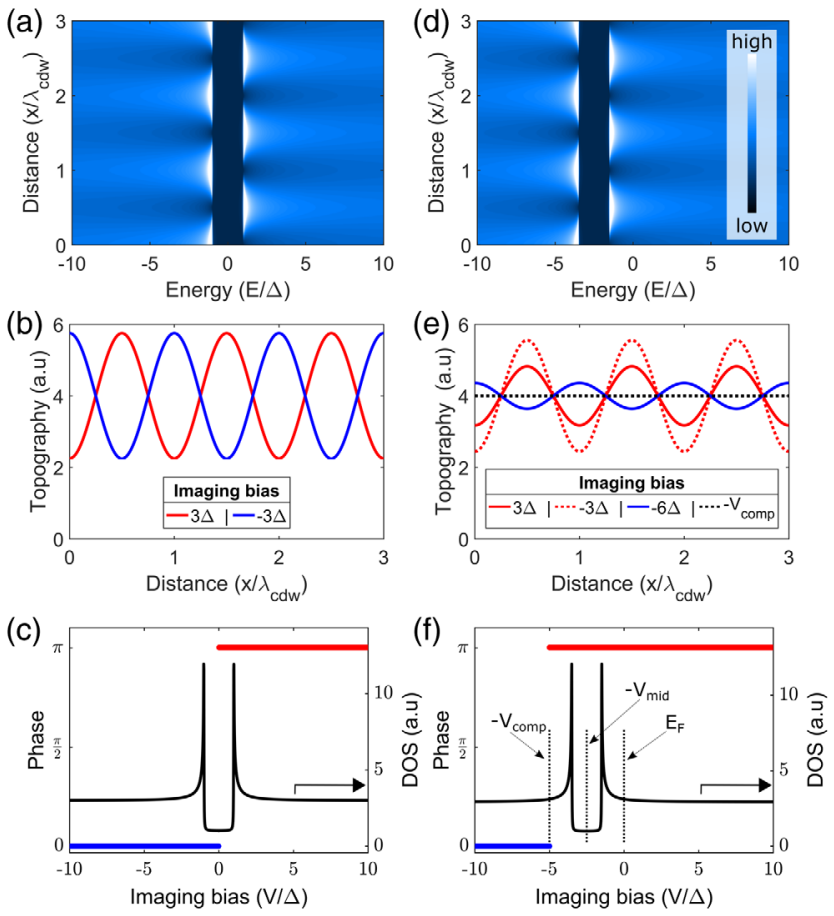

FIG. 4. One-dimensional model description of the CDW contribution to the STM topography. (a) Spatial and energy dependent CDW local DOS with the CDW gap centered at $E_{F}$. (b) Corresponding topography traces with inverted contrast between opposite polarity traces, and (c) their bias dependent phases: contrast inversion happens at $E_{F}$. (d) Spatial and energy dependent CDW local DOS with the CDW gap shifted below $E_{F}$. (e) Corresponding topography traces at selected bias voltages and (f) their bias dependent phases: contrast inversion happens below $E_{F}$. The solid black line in panel (c) and (f) illustrate the BCS-like model DOS used in the modeling. reconstruct a bias dependent topography by integrating our model local DOS [Fig. 4(a)] between $E_{F}$ and the imaging bias at each position, including a finite thermal smearing [Fig. 4(b)]. When the CDW gap is centered on $E_{F}$ [Fig. 4(a)], the integration at positive (negative) bias runs over primarily depleted (accumulated) states. In this case, CDW CI (equivalent to a $\pi$ shift for a harmonic density profile) is expected between opposite polarity images. This can be seen explicitly as a $\pi$-phase shift at $E_{F}$ [Fig. 4(c)] in the phase as a function of imaging bias of the calculated sinusoidal CDW signal in Fig. 4(b). This situation certainly does not describe our experimental data. Indeed, in this case, one does not expect to image the same CDW pattern at positive and negative sample bias, contrary to our observations reproduced in the Figs. 1 and 2.

A very different bias dependence of the phase appears when shifting the CDW gap below the Fermi level, as illustrated in Fig. 4(f). In this case, positive bias STM imaging still involves integration over primarily depleted states as in the absence of a shift. However, imaging up to a finite negative bias corresponding to the middle of the CDW gap $\left(-V_{\text {mid }}\right)$ will still reflect depleted states [Fig. 4(d)], in contrast to the unshifted case. Hence, the CDW pattern corresponds to depleted charge regions when imaging at biases between $-V_{\text {mid }}$ and a finite positive bias. No CI is expected between opposite polarity images in the range $\pm V_{\text {mid }}$ [Fig. 4(e), solid and dotted red lines], in agreement with experiment.

Reducing the bias voltage below $-V_{\text {mid }}$, both charge depletion and charge accumulation in the local density of states contribute to the tunneling current. Within the harmonic and symmetric charge redistribution model considered here, the CDW contrast is then progressively reduced to ultimately disappear at a compensation bias voltage $-V_{\text {comp }}=-2 V_{\text {mid }}$ [Fig. 4(e), dotted black line]. One will have to set the bias voltage below $-V_{\text {comp }}$ to reveal the CDW pattern corresponding to charge accumulation. Consequently, contrast inversion does not happen at $E_{F}$, but at a negative bias $-V_{\text {comp }}$ corresponding to

$$
\int_{-V_{\text {comp }}}^{-V_{\text {mid }}} \rho(E) d E=\int_{-V_{\text {mid }}}^{0} \rho(E) d E
$$

where $\rho(E)$ is the local DOS.

Note that $-V_{\text {comp }}$ can be larger than the maximum negative bias where CDW contrast is still achievable, in which case contrast inversion cannot be observed. This is the case, for example, in $\mathrm{Cu}$ intercalated $\mathrm{TiSe}_{2}$ [27]. The actual values of $-V_{\text {mid }}$ and $V_{\text {comp }}$ will depend on the detailed material band structure.

In case the gap is centered at $E_{F}$, the CDW contrast is zero for all biases within the gap, or vanishingly small when including thermal smearing effects (see Fig. S5 in Supplemental Material [16]). The situation changes completely when the gap is shifted below the Fermi level 
[Fig. 4(f)]. As shown above, the bias range where STM imaging involves integration over primarily depleted states is no longer confined to positive biases, but extends to finite negative biases. At these negative biases, STM imaging will show the same CDW pattern as at positive bias. Remarkably, for a large enough shift of the gap, we do not only expect the same pattern but also the same CDW modulation amplitude at selected opposite polarity biases as shown in Fig. S5b in the Supplemental Material (see Sec. VI in Supplemental Material for a detailed comparison of the data with the model) [16]. Another striking feature is that the CDW amplitude will remain finite for biases inside the CDW gap when the latter is shifted below $E_{F}$. Whether the CDW gap is at $E_{F}$ or below, the CDW contrast will vanish for large enough imaging biases when the weight of the reconstructed states relative to the unreconstructed states becomes too small to resolve.

The simple 1D model introduced in Fig. 4 reproduces all the topographic CDW features observed by STM on $\mathrm{TiSe}_{2}$. Considering the bias dependent CDW modulation amplitude in a region away from any defect, we can make a rough estimate of the gap amplitude and position below $E_{F}$ (see Supplemental Material Fig. S5 [16]). For a region near the middle of Fig. 2, we find a good correspondence between the data and the 1D model for a CDW gap width of $\Delta=$ $70 \mathrm{meV}$ and a shift of $\cong 1.3 \Delta$. This result is perfectly consistent with the rigid band shift moving the CDW gap to higher binding energy as a function of electron doping observed by ARPES [30,31] and STM [27]. In our experiments, we observe local variations of the doping and associated band shift near atomic impurities, which provide a unique opportunity to verify our model predictions in a single experiment. Depending on sample bias and distance from these atomic dopants, we observe all three contrast configurations discussed in Fig. 4(e). STM images reveal CDW patterns corresponding to charge accumulation regions, to charge depletion regions and regions without contrast corresponding to imaging at $-V_{\text {comp }}$ (Fig. 2). Note that finding $\mathrm{CI}$ at a finite negative sample bias in pristine $\mathrm{TiSe}_{2}$ is consistent with electron doping due to the unavoidable Ti self-doping.

Conclusions.-The comprehensive STM study presented here provides an alternative experimental insight into the amplitude and position of the CDW gap with respect to $E_{F}$, which both are still controversial [32-37]. Analyzing the bias dependence of the CDW modulation amplitude and phase, we find compelling evidence that the gap is not pinned to the Fermi level. This directly explains the absence of CDW contrast inversion between opposite polarity STM images as would be expected in the classic Peierls description. Such insight may prove instrumental in associating unknown periodic structures in STM images with a CDW. We demonstrate that CI in $\mathrm{TiSe}_{2}$ can take place at negative bias voltages significantly away from the Fermi level, with a remarkable dependence on the local doping. We find this dependence is a direct consequence of the bands and the CDW gap shifting to higher binding energy upon electron doping. The simple model we propose further explains the absence of $\mathrm{CI}$ in $\mathrm{Cu}$ doped $\mathrm{TiSe}_{2}$ as due to the CDW gap shifting significantly below the Fermi level [27]. The doping dependent CI we observe by STM poses explicit constraints on any model description of the CDW phase transition in $\mathrm{TiSe}_{2}$. It suggests in particular that the CDW formation involves primarily electronic states away from the Fermi level, which implies that the transition cannot be driven by a particular topology of the Fermi surface. The system gains energy through the momentum dependent electron-phonon and electronelectron interactions, emphasizing the strongly correlated nature of electrons in the CDW phase of $\mathrm{TiSe}_{2}$.

This project was supported by the Swiss National Science Foundation through Div. II (Grant No. 162517). We acknowledge stimulating discussions with $\mathrm{J}$. van Wezel, B. Hildebrand, T. Jaouen, T. Gazdic, Ch. Berthod, and J. Lorenzana. We thank C. Barreteau for her help with characterizing the single crystals via transport measurements, and G. Manfrini and A. Guipet for their skillful technical assistance. C.R. designed the experiment. A. S. and M.S. performed the STM measurements. M. S., A. S., and Á. P. performed data analysis. D. B. performed the DFT simulations. Á. P. conceived the one-dimensional model. E. G. synthesized the bulk crystals. C. R., Á. P., and A. S. wrote the paper. All authors contributed to the scientific discussions and manuscript revisions.

*Corresponding author.

Christoph.Renner@unige.ch

These authors contributed equally to this work.

[1] J. A. Wilson, F. J. Disalvo, and S. Mahajan, Charge-density waves and superlattices in metallic layered transition-metal dichalcogenides, Adv. Phys. 24, 117 (1975).

[2] C. W. Chen, J. Choe, and E. Morosan, Charge density waves in strongly correlated electron systems, Rep. Prog. Phys. 79, 084505 (2016).

[3] X. T. Zhu, J. D. Guo, J. D. Zhang, and E. W. Plummer, Misconceptions associated with the origin of charge density waves, Adv. Phys. X 2, 622 (2017).

[4] P. Mallet, K. M. Zimmermann, P. Chevalier, J. Marcus, J. Y. Veuillen, and J. M. G. Rodriguez, Contrast reversal of the charge density wave STM image in purple potassium molybdenum bronze $\mathrm{K}_{0.9} \mathrm{Mo}_{6} \mathrm{O}_{17}$, Phys. Rev. B 60, 2122 (1999).

[5] P. Mallet, H. Guyot, J. Y. Veuillen, and N. Motta, Chargedensity-wave STM observation in $\eta-\mathrm{Mo}_{4} \mathrm{O}_{11}$, Phys. Rev. B 63, 165428 (2001).

[6] H. L. Edwards, D. J. Derro, A. L. Barr, J. T. Markert, and A. L. de Lozanne, Spatially Varying Energy Gap in the $\mathrm{CuO}$ Chains of $\mathrm{YBa}_{2} \mathrm{Cu}_{3} \mathrm{O}_{7-x}$ Detected by Scanning Tunneling Spectroscopy, Phys. Rev. Lett. 75, 1387 (1995).

[7] D. Stoltz, M. Bielmann, M. Bovet, L. Schlapbach, and H. Berger, Tunneling evidence for spatial location of the 
charge-density-wave induced band splitting in $1 T-\mathrm{TaSe}_{2}$ Phys. Rev. B 76, 073410 (2007).

[8] W. Sacks, D. Roditchev, and J. Klein, Voltage-dependent STM image of a charge density wave, Phys. Rev. B 57, 13118 (1998).

[9] J. Dai, E. Calleja, J. Alldredge, X. Zhu, L. Li, W. Lu, Y. Sun, T. Wolf, H. Berger, and K. McElroy, Microscopic evidence for strong periodic lattice distortion in two-dimensional charge-density wave systems, Phys. Rev. B 89, 165140 (2014).

[10] K. Rossnagel, On the origin of charge-density waves in select layered transition-metal dichalcogenides, J. Phys. Condens. Matter 23, 213001 (2011).

[11] L. P. Gor'kov, Strong electron-lattice coupling as the mechanism behind charge density wave transformations in transition-metal dichalcogenides, Phys. Rev. B 85, 165142 (2012).

[12] M. Hellgren, J. Baima, R. Bianco, M. Calandra, F. Mauri, and L. Wirtz, Critical Role of the Exchange Interaction for the Electronic Structure and Charge-Density-Wave Formation in $\mathrm{TiSe}_{2}$, Phys. Rev. Lett. 119, 176401 (2017).

[13] M. D. Watson, O. J. Clark, F. Mazzola, I. Marković, V. Sunko, T. K. Kim, K. Rossnagel, and P. D. C. King, Orbitaland $k_{z}$-Selective Hybridization of Se $4 p$ and Ti $3 d$ States in the Charge Density Wave Phase of $\mathrm{TiSe}_{2}$, Phys. Rev. Lett. 122, 076404 (2019).

[14] T. Jaouen, M. Rumo, B. Hildebrand, M.-L. Mottas, C. W. Nicholson, G. Kremer, B. Salzmann et al., Unveiling the Semimetallic Nature of $1 T-\mathrm{TiSe}_{2}$ by Doping its Charge Density Wave, arXiv:1911.06053.

[15] A. M. Novello, B. Hildebrand, A. Scarfato, C. Didiot, G. Monney, A. Ubaldini, H. Berger, D. R. Bowler, P. Aebi, and Ch. Renner, Scanning tunneling microscopy of the charge density wave in $1 T$-TiSe 2 in the presence of single atom defects, Phys. Rev. B 92, 081101(R) (2015).

[16] See Supplemental Material at http://link.aps.org/ supplemental/10.1103/PhysRevLett.125.267603 for details on experimental (crystal growth and STM measurements) and theoretical (DFT) methods, on data analysis (contrast inversion by Fourier filtering and calculation of CDW amplitude in STM), and for additional DFT, STM, and STS data and figures, which includes Refs. [17-26].

[17] F. J. Disalvo, D. E. Moncton, and J. V. Waszczak, Electronic properties and superlattice formation in the semimetal $\mathrm{TiSe}_{2}$, Phys. Rev. B 14, p. 4321 (1976).

[18] G. Kresse and J. Furthmüller, Efficient iterative schemes for ab initio total-energy calculations using a plane-wave basis set, Phys. Rev. B 54, 11169 (1996).

[19] G. Kresse and J. Hafner, Ab initio molecular dynamics for liquid metals, Phys. Rev. B 47, 558 (1993).

[20] G. Kresse and D. Joubert, From ultrasoft pseudopotentials to the projector augmented-wave method, Phys. Rev. B 59, 1758 (1999).

[21] J. P. Perdew, K. Burke, and M. Ernzerhof, Generalized Gradient Approximation Made Simple, Phys. Rev. Lett. 77, 3865 (1996).

[22] J. Tersoff and D. R. Hamann, Theory and Application for the Scanning Tunneling Microscope, Phys. Rev. Lett. 50, 1998 (1983).
[23] W. A. Hofer, Challenges and errors: Interpreting high resolution images in scanning tunneling microscopy, Prog. Surf. Sci. 71, 147 (2003).

[24] G. Grüner, Density waves in solids, in Frontiers in Physics, Advanced Book Program xxi (Addison-Wesley Pub. Co., Reading, MA, 1994), p. 259.

[25] C. J. Chen, Introduction to Scanning Tunneling Microscopy, Oxford Series in Optical and Imaging Sciencesxxii (New York, Oxford University Press 1993), p. 412, p. 31 of plates.

[26] A. M. Novello, M. Spera, A. Scarfato, A. Ubaldini, E. Giannini, D. R. Bowler, and Ch. Renner, Stripe and Short Range Order in the Charge Density Wave of $1 T-\mathrm{Cu}_{x} \mathrm{TiSe}_{2}$, Phys. Rev. Lett. 118, 017002 (2017).

[27] M. Spera, A. Scarfato, E. Giannini, and Ch. Renner, Energydependent spatial texturing of charge order in $1 T-\mathrm{Cu}_{x} \mathrm{TiSe}_{2}$, Phys. Rev. B 99, 155133 (2019).

[28] C. J. Arguello, S. P. Chockalingam, E. P. Rosenthal, L. Zhao, C. Gutierrez, J. H. Kang, W. C. Chung et al., Visualizing the charge density wave transition in $2 \mathrm{H}-\mathrm{NbSe}_{2}$ in real space, Phys. Rev. B 89, 235115 (2014).

[29] J. Hall, N. Ehlen, J. Berges, E. van Loon, C. van Efferen, C. Murray, M. Rosner et al., Environmental control of charge density wave order in monolayer $2 \mathrm{H}-\mathrm{TaS}_{2}$, ACS Nano 13, 10210 (2019).

[30] T. Rohwer, S. Hellmann, M. Wiesenmayer, C. Sohrt, A. Stange, B. Slomski, A. Carr et al., Collapse of long-range charge order tracked by time-resolved photoemission at high momenta, Nature (London) 471, 490 (2011).

[31] M. L. Mottas, T. Jaouen, B. Hildebrand, M. Rumo, F. Vanini, E. Razzoli, E. Giannini et al., Semimetal-tosemiconductor transition and charge-density-wave suppression in $1 T-\mathrm{TiSe}_{2-x} S_{x}$ single crystals, Phys. Rev. B 99, 155103 (2019).

[32] R. V. Coleman, Z. Dai, W. W. McNairy, C. G. Slough, and W. C., STM \& AFM of layered transition metal compounds, in Surface Properties of Layered Structures, Physics and Chemistry of Materials with Low-Dimensional Structures, edited by G. Benedek (Springer, Dordrecht, 1992).

[33] M. Iavarone, R. Di Capua, X. Zhang, M. Golalikhani, S. A. Moore, and G. Karapetrov, Evolution of the charge density wave state in $\mathrm{Cu}_{x} \mathrm{TiSe}_{2}$ Phys. Rev. B 85, 155103 (2012).

[34] S. Yan, D. Iaia, E. Morosan, E. Fradkin, P. Abbamonte, and V. Madhavan, Influence of Domain Walls in the Incommensurate Charge Density Wave State of $\mathrm{Cu}$ Intercalated $1 T$-TiSe 2 Phys. Rev. Lett. 118, 106405 (2017).

[35] B. Hildebrand, T. Jaouen, C. Didiot, E. Razzoli, G. Monney, M. L. Mottas, F. Vanini et al., Local resilience of the $1 T$-TiSe ${ }_{2}$ charge density wave to Ti self-doping, Phys. Rev. B 95, 081104 (2017).

[36] K.-W. Zhang, C.-L. Yang, B. Lei, P. Lu, X.-B. Li, Z.-Y. Jia, Y.-H. Song et al., Unveiling the charge density wave inhomogeneity and pseudogap state in $1 T-\mathrm{TiSe}_{2}$, Sci. Bull. 63, 426 (2018).

[37] S. Kolekar, M. Bonilla, Y. Ma, H. C. Diaz, and M. Batzill, Layer- and substrate-dependent charge density wave criticality in 1T-TiSe 2 2D Mater. 5, 015006 (2017). 